\title{
CIRURGIA DE CATARATA PELA TÉCNICA DE FACOEMULSIFICAÇÃO: UM ESTUDO DE CASO
}

\author{
Andréia Fiorini de Assis CARVALHO ${ }^{1}$ \\ Roberta Bessa Veloso SILVA² \\ Eric Batista FERREIRA ${ }^{3}$
}

\begin{abstract}
${ }^{1}$ Acadêmica do curso de Medicina, Universidade José do Rosário Vellano/UNIFENAS e-mail: deiafiorini@msn.com ${ }^{2}$ Doutora em Estatística e Experimentação Agropecuária/UFLA, Universidade José do Rosário Vellano - UNIFENAS ${ }^{3}$ Doutor em Estatística e Experimentação Agropecuária/UFLA, Universidade Federal de Alfenas - UNIFAL
\end{abstract}

Recebido em: 03/03/2016 - Aprovado em: 21/05/2016 - Disponibilizado em: 30/07/2016

\begin{abstract}
RESUMO: A catarata é uma enfermidade ocular grave provocada pela opacificação do cristalino que é uma lente natural do olho responsável pela focalização da luz sobre a retina. Esta enfermidade pode ocorrer em razão da idade, fator genético, traumas e doenças. A perda da visão impede o indivíduo de realizar suas atividades diárias, além da dificuldade de reconhecer amigos e familiares. Com o decorrer do tempo, a catarata pode se agravar culminando na cegueira reversível. Dentre as diversas causas, a velhice é a mais comum e o principal sinal é a redução da acuidade visual. Para identificar o perfil dos pacientes com catarata que foram submetidos à cirurgia pela técnica de facoemulsificação foi realizado um estudo transversal, de base secundária, na Clínica de Olhos Visiocentro, situada na cidade de Campo Belo, MG. Foram analisados 1033 registros médicos no período de janeiro a dezembro dos anos de 2014 e 2015. Os resultados permitiram concluir que a faixa etária prevalente de cirurgias foi de 70 a 79 anos, sendo a maioria do gênero feminino. As frequências de cirurgias realizadas no olho direito foram semelhantes àquelas encontradas para o olho esquerdo. Verificou-se que os pacientes utilizam de recursos próprios (particular) para financiarem a cirurgia.
\end{abstract}

Palavras-chave: Qualidade de vida, Perda da visão, Lateralidade dos olhos, oftalmologia.

\begin{abstract}
Cataract is a serious eye disease caused by clouding of the natural lens of the eye responsible for focusing light on the retina. This disorder can occur due to age, genetic factors, trauma and diseases. The loss of vision prevents an individual from performing daily activities, despite the difficulty in recognizing friends and family. Along time, the cataract may culminate in blindness. Among the various causes, old age is the most common and the main way, is the reduction of visual acuity. To identify the profile of patients with cataract who underwent surgery by phacoemulsification was a cross-sectional study, a secondary basis in the clinic Olhos Visiocentro, located in Campo Belo, Brazil. Records of 1033 patients were analyzed from January 2014 to December 2015. Results showed that the prevalent age of surgery was 70 to 79 years, most of them female. The frequency of surgeries performed in the right eye were similar to those found for the left eye. It was found that most patients used own financial resources for the surgery. Keywords: Life quality, loss of vision, eyes laterality, ophthalmology.
\end{abstract}

\section{INTRODUÇÃO}

A catarata é uma enfermidade ocular grave provocada pela opacificação do cristalino que é uma lente natural do olho responsável pela focalização da luz sobre a retina. Quando o cristalino fica opaco, a luz não chega à retina na quantidade satisfatória e perturba a qualidade da visão, que é tida como o principal sentido do ser humano. Esta enfermidade pode ocorrer em razão da idade, fator genético, traumas e doenças.

A perda da visão impede o indivíduo de ser capaz de dirigir, de realizar suas atividades diárias, além da dificuldade de reconhecer amigos e familiares. Com o decorrer do tempo, a catarata pode se agravar culminando na cegueira reversível. Dentre as diversas causas, a velhice é a mais comum e, o principal sintoma, é a redução da acuidade visual. 
A cegueira por catarata aumenta a dependência do indivíduo, torna-o incapacitado, e diminui sua condição social, acarretando problemas psicossociais $\mathrm{e}$ econômicos. Para a sociedade, simboliza um encargo oneroso e redução da força laboral, pois acarreta a perda da autoestima, limitações ocupacionais e, por conseguinte, redução da renda familiar.

A alternativa para a recuperação da visão é através da cirurgia de catarata, que gera benefícios, como a volta a capacidade laboral, econômico e social para o indivíduo, para a sua família e toda a comunidade. A cirurgia de catarata representa não apenas a recuperação da transparência dos meios oculares, mas também é um método cirúrgico apropriado para corrigir os erros refrativos devido a algumas anomalias ópticas diagnosticadas no pré-operatório.

Dessa maneira, este trabalho teve como objetivo caracterizar o perfil dos pacientes encaminhados para cirurgia de facoemulsificação, em uma clínica de olhos na cidade de Campo Belo, $\mathrm{MG}$, sob a supervisão dos médicos oftalmologistas Dr. Alisson de Assis Carvalho e Dr. Fernando Barbosa Vilela.

\section{A CATARATA}

De acordo com Dantas (2013), o olho humano possui em média $24,4 \mathrm{~mm}$ de diâmetro e é partilhado em dois segmentos: anterior e posterior. $\mathrm{O}$ segmento anterior envolve a córnea, a íris, o corpo ciliar e o cristalino, sendo preenchido pelo humor aquoso que nutre as composições internas do olho e conservam a sua pressão.

O segmento posterior vai da parte posterior do cristalino até a retina e inclui o humor vítreo. A medida do cristalino varia entre 0,7 e $0,9 \mathrm{~mm}$ de comprimento no seu maior eixo e, entre 0,2 e $0,4 \mathrm{~mm}$ de espessura. O cristalino não para de crescer ao longo da vida, ou seja, está em contínuo desenvolvimento (DANTAS, 2013).

Segundo Rezende (2010), no olho jovem, o cristalino é transparente, conduz a luz e tem a capacidade de mudar a sua forma (convexidade maior ou menor) conforme a distância do objeto, fato conhecido como acomodação.

Com avançar dos anos, essa capacidade fica depreciada pelo aumento de rigidez dessa estrutura. Essa perda da capacidade de acomodação é chamada de presbiopia. Além desse problema, o cristalino pode se tornar opaco, esbranquiçado ou amarelado interferindo na capacidade de transmitir os raios luminosos provindos da córnea até a retina. Tal opacificação do cristalino é conhecida como catarata (REZENDE, 2010).

Conforme Lima Filho (2014), a catarata é a opacificação do cristalino, que acontece por uma desnaturação das proteínas que formam o cristalino e provoca redução da acuidade visual e, nos casos mais avançados, a cegueira. Pode ocorrer em um ou em ambos os olhos, porém, na maioria dos casos, desenvolve de forma simétrica em ambos os olhos.

A catarata pode ser classificada, conforme a sua localização, sendo: nuclear, cortical, subescapular e polar. A catarata nuclear, responsável pela diminuição da visão de longe, a mais típica em pacientes em idade senil, é marcada pela elevação da densidade do núcleo do cristalino (NEURAUTER, 2008). Nas Figuras 1 e 2 estão ilustrados o cristalino e a catarata nuclear.

Figura 1: Cristalino

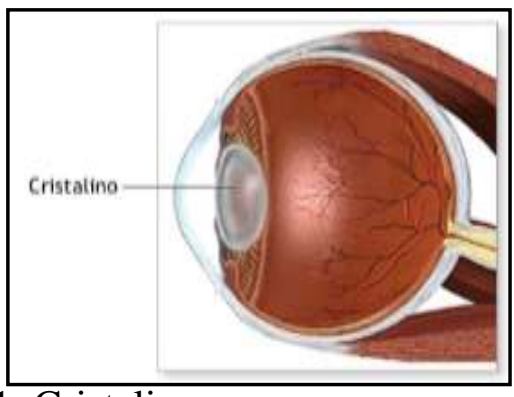

Fonte: Adaptado de Correia (2013). 


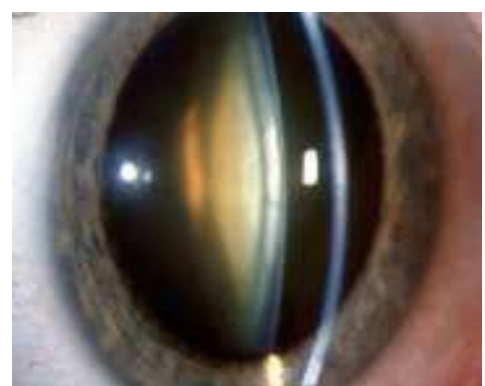

Figura 2: Catarata nuclear

Fonte: Adaptado de HORN (2016).

De acordo com Coelho (2007), o nível de opacidade de uma catarata está de forma direta associada à porcentagem do reflexo tapetal que ela impede. Conforme o grau de opacidade, a catarata se classifica em incipiente, imatura, madura, hipermadura e morganiana.

Soriano (2004) define que a catarata incipiente se constitui o estágio inicial, com opacidade focal, que atinge menos de 10 a $15 \%$ do espaço do cristalino, não havendo comprometimento do reflexo tapetal. Ela comumente afeta a região cortical, subcapsular ou capsular e, conforme a causa, pode ou não avançar. Contudo, a catarata imatura compõe o estágio intermediário, que mostra opacidade um pouco mais evidente, com comprometimento parcial do reflexo tapetal. Já a catarata madura, revela a opacidade total, com bloqueio total do reflexo tapetal.

Nas Figuras 3 e 4 estão ilustrados os estágios evolutivos de cataratas imatura e madura, respectivamente, conforme Medgrupo (2012).

Figura 3: Catarata imatura

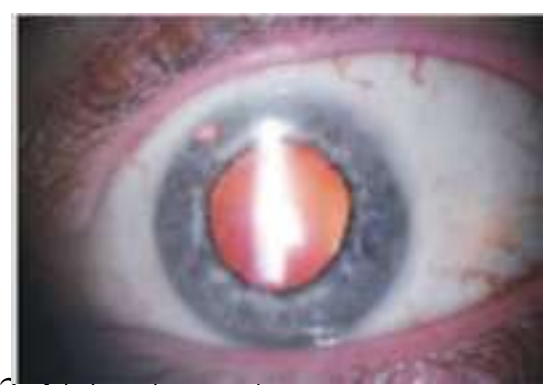

Fonte: Adaptado de Medgrupo (2012)

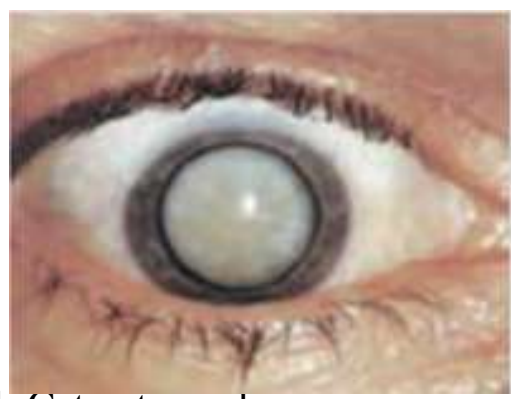

Figura 4: Catarata madura

Fonte: Adaptado de Medgrupo (2012)

Segundo Medgrupo (2012), a catarata hipermadura é o último estágio do progresso da enfermidade, havendo comprometimento das cápsulas anterior e posterior do córtex e do núcleo e, neste estágio, pode-se observar que seu aspecto é cintilante e o reflexo tapetal vai se alterar em virtude do nível de reabsorção.

No estágio da catarata morganiana, o córtex está totalmente liquefeito e o núcleo desprendido dentro da cápsula. As Figuras 5 e 6 ilustram os estágios de cataratas hipermadura e morganiana, repectivamente.

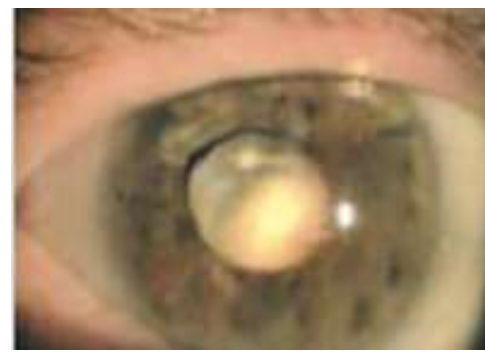

Figura 5: Catarata hipermadura

Fonte: Adaptado de Medgrupo (2012)

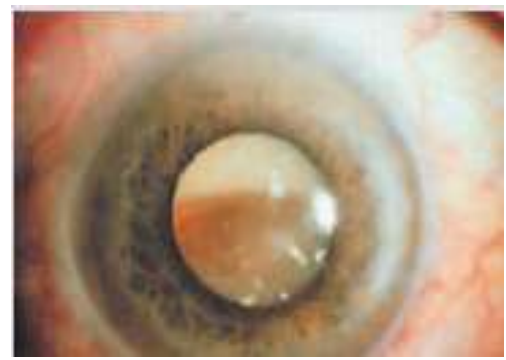

Figura 6: Catarata morganiana

Fonte: Adaptado de Medgrupo (2012)

As causas da catarata podem ser classificadas em congênita, senil, traumática, por enfermidades e medicamentosa. A catarata congênita pode resultar das malformações oculares, infecções 
intrauterinas, síndromes genéticas, mudanças sistêmicas com falhas inatas do metabolismo, hereditariedade, uso de fármacos, radiação ou ser idiopática, (OLIVEIRA et al., 2004).

No Brasil, a rubéola congênita é uma das responsáveis pela catarata e, diante disso, foi implementado o "teste do olhinho", com a finalidade de detectar possíveis anormalidades (OLIVEIRA et al., 2004).

A catarata senil, de acordo com Kanski (2004), é provocada pela idade avançada do indivíduo, porém não existe uma explicação clara sobre o motivo da opacidade em decorrência da idade, mas acredita-se que os responsáveis sejam os radicais livres. Já a catarata traumática é causada por acidentes, geralmente ela é unilateral, ou seja, acomete apenas um olho.

Em se tratando da catarata causada por doenças, o diabetes é o principal responsável. Nesta circunstância a doença surge de forma precoce e a perda visual pode ser mais acelerada. No diabetes, o cristalino pode ficar super hidratado. Neste caso, opacidades podem se originar na parte frontal ou posterior do cristalino, surgindo assim, a catarata. Há casos, em que a catarata por diabetes pode se estabelecer durante alguns dias. Já a catarata medicamentosa é resultante do uso de medicamentos, como corticoides, na forma oral ou em colírio por um período prolongado (KANSKI, 2004).

Conforme Brasil (2008), a catarata afeta ambos os sexos, porém de acordo com estudos da Organização Mundial da Saúde, as mulheres são as mais afetadas.

Fornazari (2009), coordenadora do Núcleo de Prevenção à Cegueira da Universidade Estadual de Campinas, explicou que isso pode ser devido a questões hormonais e uso de contraceptivos.

De acordo com Coelho (2007), um dos primeiros sintomas da catarata é a percepção da perda progressiva da qualidade visual. Em alguns períodos, a visão fica mais embaçada e os objetos podem parecer amarelados ou distorcidos. Normalmente, as pessoas sentem que precisam de mais iluminação para enxergar melhor e, mesmo utilizando-se de óculos, a visão permanece embaçada.
Conforme está apresentado na Figura 7 , à medida que a doença progride, pode ser notada no centro da pupila, uma mancha branca ou amarelada. E, geralmente, a pessoa só começa a notá-la em estágios mais avançados. Isso pode ser evitado através de consultas ao oftalmologista que será capaz de identificar a catarata e, então adotar o tratamento adequado.
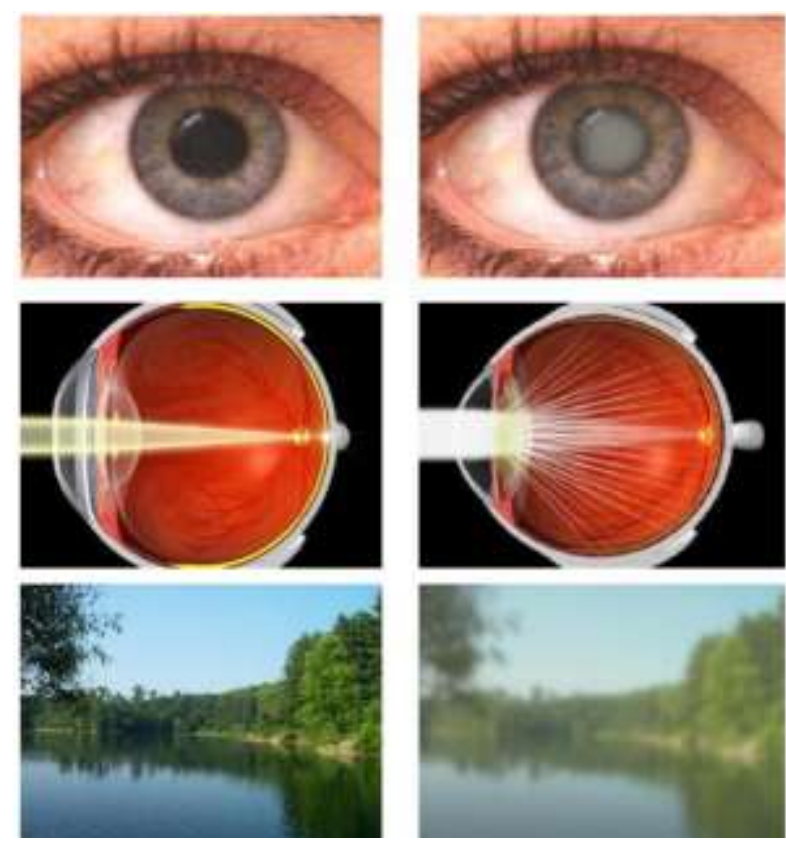

Figura 7: Percepção da visão com catarata

Fonte: Adaptado de HORN (2016).

Os sinais detectados no exame oftalmológico de rotina são a perda da acuidade visual, sendo utilizada a Tabela de Snellen para mensurar a mudança na transparência do cristalino, e a biomicroscopia do segmento anterior em midríase através do teste feito com lâmpada de fenda. Deve-se associar a queixa pessoal do paciente aos sinais objetivos do exame oftalmológico. As queixas mais comuns são redução da acuidade visual, visão "nublada", sensibilidade maior à luz, mudança da visão de cores, alteração frequente da refração (ORÉFICE; BORATTO, 2001).

Segundo Coelho (2007), o tratamento da catarata é feito por cirurgia que incide na remoção do cristalino opacificado por meio de duas técnicas utilizadas que são a extração extracapsular (EEC) e a facoemulsificação. 
Na técnica de extração extracapsular a incisão é de $8 \mathrm{~mm}$ a $10 \mathrm{~mm}$ na área limbar ou escleral por onde o núcleo do cristalino é retirado por inteiro e o material cortical é aspirado, conservando a cápsula posterior intacta. Após remoção da catarata é inserida a lente intraocular e feita a sutura da incisão. Este método é utilizado até os dias de hoje, especialmente em serviços públicos de saúde por universitários, em razão dos baixos custos de sua execução, da reduzida necessidade de habilidades e treinamento do cirurgião que a executa.

De acordo com Lima Filho (2014), a cirurgia de catarata, pelo método de facoemulsificação, é uma cirurgia ambulatorial que dura em média 19 minutos, sendo o procedimento cirúrgico mais feito nos países desenvolvidos. A terapêutica cirúrgica leva a reduções de gastos com a saúde e sociais a longo prazo, além de apresentar melhores resultados de acuidade visual e índices reduzidos de complicações cirúrgicas em comparação com a técnica de extração extracapsular (EEC).

No método por facoemulsificação o cristalino é retirado em porções fragmentadas e emulsificadas. Nesta técnica são feitas duas incisões na córnea que variam de $1,5 \mathrm{~mm}$ a $3,2 \mathrm{~mm}$, conforme a lente intraocular utilizada, que é por onde se introduz a ponteira da caneta de facoemulsificação que vibra a uma frequência ultrassônica realizando a emulsificação e aspiração do cristalino (COELHO, 2007).

Nas Figuras 8 e 9 estão apresentadas, respectivamente, a técnica de extração da catarata pela facoemulsificação e a lente intraocular inserida após o procedimento.

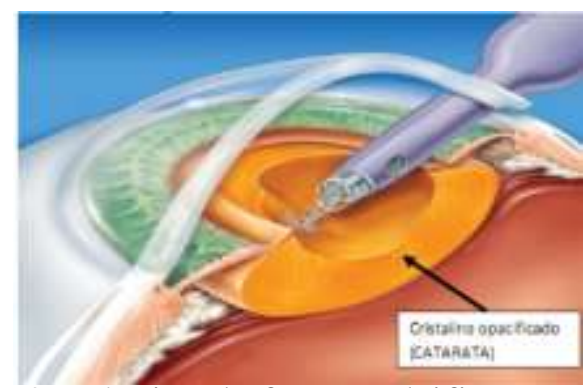

Figura 8: Técnica de facoemulsificação Fonte: Adaptado de Luz (2013)

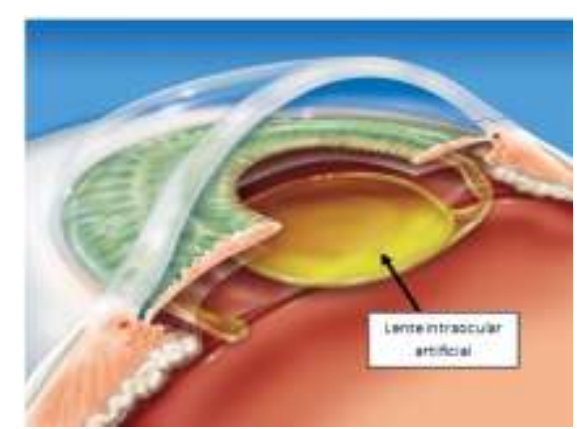

Figura 9: Lente intraocular

Fonte Adaptado de Luz (2013)

Conforme Silveira (2004), a cirurgia de catarata representa, não apenas a recuperação da transparência dos meios oculares, mas é também um método cirúrgico apropriado para corrigir os erros refrativos e algumas anomalias ópticas diagnosticadas no pré-operatório. Cabe lembrar que nas nações desenvolvidas, por possuir um acesso mais fácil a assistência oftalmológica, não há acúmulo indivíduos com cegueira por catarata.

No Brasil, como nas demais nações em desenvolvimento, o problema toma proporções alarmantes pois, junto ao número de indivíduos cegos em razão da catarata desassistidos ao longo do tempo, soma-se um contingente anual de casos novos que é superior à capacidade dos serviços de saúde existentes até 1998, (SILVEIRA, 2004).

De tal modo, percebe-se que nas nações em desenvolvimento o acesso a tais serviços é problemático. Assim o Ministério da Saúde projetou novas estratégias para expandir a assistência oftalmológica, criando em 1998 a Campanha Nacional de Cirurgias Eletivas Cirurgia de Catarata, conhecido como o Mutirão de Catarata (SILVEIRA, 2004).

\section{METODOLOGIA}

Foi conduzido um estudo transversal, com base em dados secundários, na Clínica de Olhos Visiocentro, na cidade de Campo Belo, MG. De acordo com o IBGE (2015), a cidade de Campo Belo possui aproximadamente 54.076 habitantes e está localizada na região sul de Minas Gerais.

A população-alvo em estudo foram todos registros médicos de pacientes 
encaminhados para cirurgia de facoemulsificação, atendidos na Clínica de Olhos Visiocentro, nos anos de 2014 e 2015. Para tanto, foram coletados 1033 dados referentes ao período de janeiro a dezembro dos anos de 2014 e 2015.

As informações coletadas foram referentes ao gênero, cidade de origem, lateralidade dos olhos em que a cirurgia foi realizada, convênios (SUS, particular, outros) e faixa etária.

Após a coleta das informações, os dados foram organizados através dos gráficos de colunas e de setores, sendo obtidas as frequências absoluta, relativa e percentual. Para a elaboração dos gráficos foi utilizado o software Excel (2010).

Em seguida foi utilizado um teste para proporção, ao nivel nominal de $5 \%$ de significância, para garantir com $95 \%$ a confiabilidade dos resultados.

\section{RESULTADOS E DISCUSSÃO}

De acordo com os dados obtidos, pode-se observar-se que foram realizadas, em média, 43 cirurgias de catarata por mês. Podese verificar que $59 \%$ dos pacientes encaminhados para cirurgia são do gênero feminino e $41 \%$ do gênero masculino., conforme está apresentado na Figura 10.

Nota-se uma superioridade de 18 pontos percentuais em relação ao gênero masculino, representando uma frequência de 1,4 vezes maior de cirurgias realizadas em mulheres.

Estes resultados corroboram com os obtidos por Fornazari (2009) que observou que a prevalência de catarata foi maior nas mulheres. Isso se deve a fatores hormonais que podem contribuir para o surgimento da catarata.

Brasil (2008) também observou que a catarata afeta ambos os sexos, porém de acordo com estudos da Organização Mundial da Saúde, as mulheres são as mais afetadas.

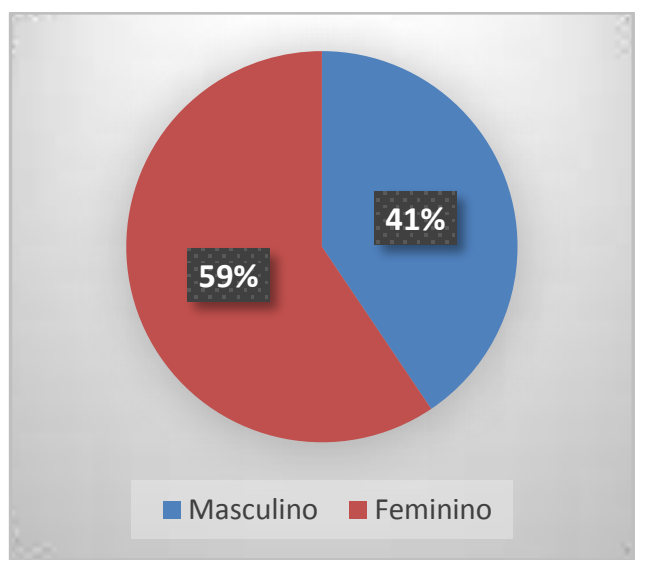

Figura 10. Distribuição de gêneros.

Fonte: Autores.

De acordo com o teste para proporção verificou-se que existe diferença significativa entre o gênero feminino e masculino, em relação à frequência de cirurgias de catarata $(\mathrm{p}<0,01)$.

Na Figura 11 observa-se que a maior frequência de cirurgias de catarata é realizada em pacientes na faixa de 70 a 79 anos. Porém, deve-se ressaltar que a prevalência de cirurgias de catarata nas faixas etárias de 60 a 69 anos e 80 a 89 totalizaram $46 \%$.

Resultados semelhantes foram obtidos por Kanski (2004) ao relatar que a catarata senil é provocada pela idade avançada do indivíduo.

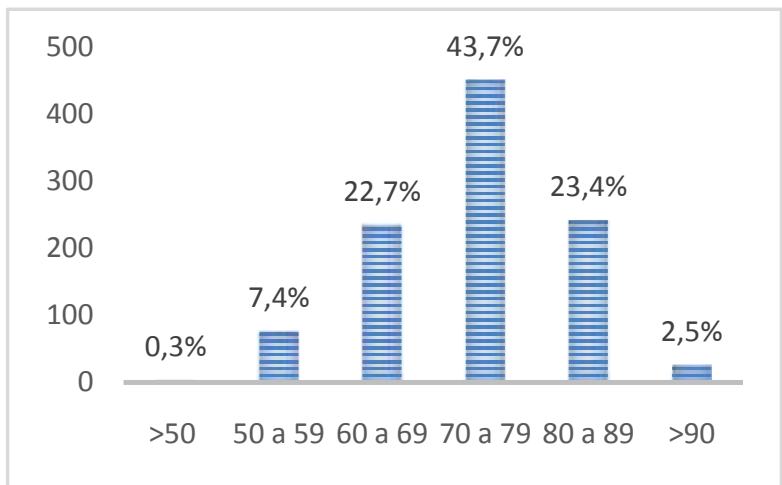

Figura 11. Distribuição etária.

Fonte: Autores.

Porém, não existe explicação explícita sobre o motivo da opacidade em decorrência da idade, mas acredita-se que os responsáveis sejam os radicais livres.

Outra característica que pode ser observada na Figura 12 foi que 50,2\% dos 
pacientes realizam a cirurgia de catarata por meio de financiamento próprio (particular). Destaca-se que 33,2\% utilizam os recursos do Sistema Único de Saúde (SUS), ou seja, 1,5 vezes menor se comparado ao uso de recursos próprios. O restante dos pacientes, 16,6\%, usam outros tipos de recursos.

Este resultado pode ser comparado com àquele obtido pelo Ministério da Saúde em 1998, que iniciou um programa para incentiar a realização de cirugias de cataratas. Este estudo mostrou que a maioria dos pacientes utiliza recursos particulares para realiar a cirurgia, mesmo tendo a opção de utilzar recursos do Sistema Único de Saude (SUS).

Apesar das 50,2\% utilizarem recursos próprios e 33,2\%, o SUS para financiar a cirurugia, não foi evidenciado diferença significativa entre as proporões, $(\mathrm{p}=0,3113)$.

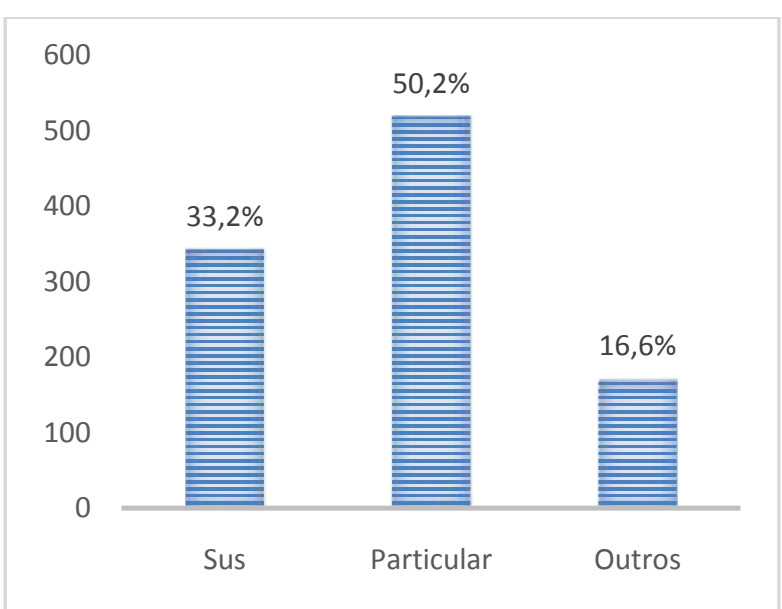

Figura 12. Recursos utilizados pelos pacientes para a realização da cirurgia de catarata.

Fonte: Autores.

Pode-se verficar, na Figura 13, que as frequências de cirurgias realizadas no olho direito $(49,2 \%)$ e olho esquerdo $(50,3 \%)$ foram muito semelhantes. Apenas uma parcela pequena de pacientes $(0,5 \%)$ realiza a cirurgia em ambos os olhos.

Este resultado foi semelhante ao obtido por Lima Filho (2014), que verificou que a catarata pode ocorrer em um ou em ambos os olhos e que, na maioria dos casos, desenvolve de forma simétrica em ambos os olhos.
Esta pequena parcela da população que faz cirurgia em ambos os olhos indica que mesmo diante de todos os sintomas da catarata estes deixaram evoluir para o estágio de cegueira reversível.

O resultado do teste para proporção, ao nível nominal de 5\% de significância, confirmou que não houve diferença nas cirurgias realizadas no olho direito e olho esquerdo, $(p=0,6284)$.

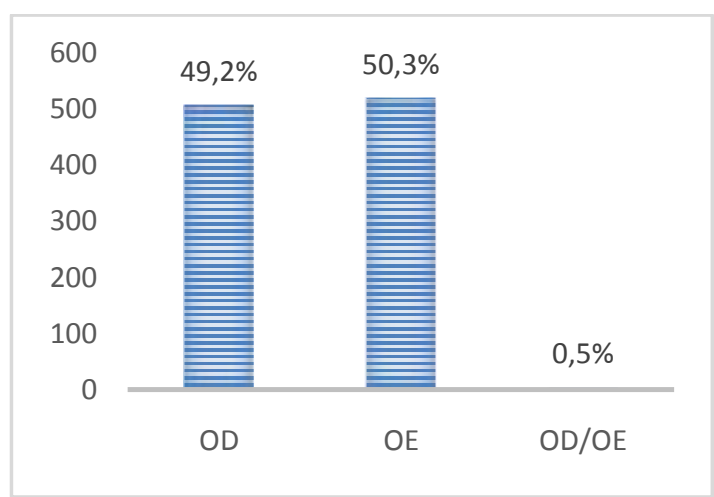

Figura 13. Lateralidade da cirurgia.

Fonte: Autores.

\section{CONCLUSÕES}

Pode-se concluir que a maioria dos pacientes registrados na Clínica de Olhos Visiocentro são do gênero feminino e residentes na cidade de Campo Belo, MG. A faixa etária prevalente de cirurigas de catarata foi de 70 a 79 anos, sendo utilizados os recursos próprios e do SUS para financiar a cirurgia.

Verificou-se semelhança entre a frequência de cirurgias feitas na lateralidade dos olhos, ou seja, a frequência de cirurgias realizadas no olho direito foi praticamente igual à frequência de ocorrência no olho esquerdo.

Observou-se uma tendência da população de idosos com catarata procurarem cada vez mais a realização da cirurgia de catarata por facoemulsificação assim que os primeiros sintomas aparecem para melhoria da qualidade de vida senil. 


\section{REFERÊNCIAS BIBLIOGRÁFICAS}

BRASIL. Ministério da Saúde. Secretaria de Atenção à Saúde. Departamento de Atenção Especializada. Relatório de gestão: atenção especializada média e alta complexidade 2000-2005. Brasília: Ministério da Saúde, 2008.

COELHO, R.P. Catarata e outras doenças do cristalino. In: BICAS Harley; JORGE André. Oftalmologia: fundamentos e aplicações. São Paulo: Tecmedd, 2007. p. 293-299.

CORREIA, J.S. Saúde! Cristalino artificial. 2013. Disponível em: http://saudejoni.blogspot.com.br/2013/02/cristalinoartificial.html. Acesso em: 03 mar. 2016.

DANTAS, A.M. Anatomia do Aparelho Visual. In: Série Oftalmologia Brasileira (CBO) 3.ed. São Paulo: Guanabara Koogan, 2013. P.514-526.

IBGE - INSTITUTO BRASILEIRO DE GEOGRAFIA E ESTATÍSTICA. Cidades Campo Belo/MG. Disponível em:

http://www.cidades.ibge.gov.br/xtras/perfil.ph $\mathrm{p}$ ?lang $=\&$ codmun $=311120$. Acesso em: 29 jan. 2016

HORN - Hospital de Olhos do RN. Doenças oculares. Disponível em:

http://www.hospitaldeolhosdorn.com.br/probl emas_oculares/catarata.htm. Acesso em 03 mar. 2016.

KANSKI, J. Oftalmologia clínica. Rio de Janeiro: Elsevier, 2004.

LIMA FILHO, A.A. Bases da Oftalmologia

I. Coleção Conselho Brasileiro de Oftalmologia. 3.ed. Rio de Janeiro: Guanabara, 2014.

LUZ, R. A. Vigilância epidemiológica de endoftalmite e Síndrome Tóxica do Segmento Anterior após cirurgias de catarata: identificação e seleção de marcadores. 2013, 105 p. Dissertação [Mestrado em Enfermagem]. Universidade de São Paulo, São Paulo, 2013.

MEDGRUPO. Oftamolgia. Rio de Janeiro: Medyin, 2012.

NEURAUTER R. Indicações e contraindicações na cirurgia de catarata. In: PADILHA, M.A. Catarata. 2 ed. Rio de Janeiro: Cultura Médica; 2008. p. 47- 53.

OLIVEIRA, M.L.S.; DI GIOVANNI, M.E.; PORFIRIO NETO JR, F.; TARTARELLA, M.B. Catarata congênita: aspectos diagnósticos, clínicos, e cirúrgicos em pacientes submetidos a lensectomia. Arquivos Brasileiros de Oftalmologia. 2004; v.67, n.6, p. 921-926.

ORÉFICE, F.; BORATTO; L.

Biomicroscopia do cristalino. In: ORÉFICE, F.; BORATTO L. Biomicroscopia e gonioscopia. 2 ed . Rio de Janeiro: Cultura Médica; 2001. p.72-80.

FORNAZARI, D. A Cegueira poderia ser evitada em 75\% dos casos. Publicado em: 9 out. 2009. Disponível em:

http://bvsms.saude.gov.br/bvs/sus/pdf/outubro loms_cegueira_poderia_evitada_0910.pdf. Acesso em: 29 jan. 2016

REZENDE, F. Cirurgia de catarata. 3.ed. Rio de Janeiro: Cultura Médica, 2010.

SILVEIRA, C.C.A. Mutirão de Catarata: uma estratégia nacional de atenção á saúde. 2004, 111 p. Dissertação [Mestrado em Gestão de Sistemas e Serviços da Saúde] Fundação Oswaldo Cruz, Rio de Janeiro, 2004.

SORIANO, E.S. Catarata e alterações do cristalino. In: SCHOR Paulo; CHAMON, Wallace; BELFORT JÚNIOR, Rubens. Oftalmologia. São Paulo: Manole; 2004. p.269-275. 